УДК 3179

\title{
АНАЛИЗ ЭВОЛЮЦИИ СЛОЖНЫХ СИСТЕМ МЕТОДОМ СТРУКТУРНО-ФАЗОВЫХ ПЕРЕХОДОВ
}

\author{
Иванов О.П. \\ в.н.с., к.геол-мин.н. \\ Музей Землеведения МГУ \\ им. М.В. Ломоносова
}

\begin{abstract}
Аннотация: Учитывая, что логистическое уравнение Ферхюльста является нелинейным динамическим, для него проводится анализ зон бифуркаций, названных автором структурно-фазовыми переходами для сложных систем, с помощью универсального алгоритма. Здесь по М.Файгенбауму, возникают переходы от упорядочения к хаосу. На основе введения понятий адаптивности и самоподобия строится матрица по алгоритму самоорганизации устойчивых структур. Она позволяет проводить сравнение величины отношений управляющих параметров в точках бифуркаций со значениями корней обобщенного уранения золотых сечений. Это позволяет оценивать степень адапттивности. Достоверность подхода показана на примерах усталостного разрушения образцов из сплавов при циклических нагрузках и в случае кристаллизации структур из капли концентрированного раствора с участтием дисперсных порошков. Показано, что алгоритм удобен как при диссипативных процессах, так и в варианте кумулятивных процессов.
\end{abstract}

\section{ANALYSIS OF THE EVOLUTION OF COMPLEX SYSTEMS BY THE METHOD OF STRUCTURAL-PHASE TRANSITIONS}

\section{Ivanov O.P.}

\begin{abstract}
Given that the Verhulst logistic equation is a nonlinear dynamic one, the analysis of bifurcation zones, called structural-phase transitions for complex systems by the author, is carried out using a universal algorithm. Here, according to M. Feigenbaum, there is a transition from order to chaos. Based on the introduction of the concepts of adaptivity and self-similarity, a matrix is constructed according to the algorithm of self-organization of stable structures. It allows us to compare the
\end{abstract}


value of the relations of the control parameters at the bifurcation points with the values of the roots of the generalized uranization of the golden sections. This allows you to evaluate the degree of adaptability. The reliability is shown by examples of fatigue failure of alloy samples under cyclic loads and in the case of crystallization of structures from a drop of a concentrated solution with the appearance of dispersed powders. It is shown that the algorithm is convenient both for dissipative processes and for cumulative processes.

Обсуждение. Задача превентивной защиты социума от опасных природных процессов актуальна. Развитие таких процессов до экстремальных состояний проходит через последовательность перестроечных фаз (зон бифуркаций), в которых меняются механизмы изменения энергетики. Они названы нами структурно-фазовыми переходами сложных систем. Предлагается универсальный алгоритм, позволяющий исследовать специфику таких фаз и топологию их распределения на диаграммах эволюции, что позволит в дальнейшем выбирать «акупунктурные точки» для превентивных воздействий.

В этой связи обратим внимание на квазициклическое логистическое уравнения Ферхюльста: $\mathrm{x}_{\mathrm{n}+1}=\lambda \mathrm{x}_{\mathrm{n}}\left(1-\mathrm{x}_{\mathrm{n}}\right)$

По М.Файгенбауму поведение таких хаотических систем отвечает сценарию удвоения периода перехода к хаотической динамике Т - 2Т - 4T -..., Предельная скорость перехода определяется константой $\delta=4,669$. Характерно, что переходы в точках бифуркации $\mathrm{x}_{\mathrm{n}}{ }^{*}$ носят самоуправляемый характер, так как адаптация контролируется обратной связью. Одновременно здесь реализуется условие автомодельности: $\mathrm{x}_{\mathrm{n}} * / \mathrm{x}_{\mathrm{n}+1} *=(1 / \lambda *) 1 /\left(1-\mathrm{x}_{\mathrm{n}} *\right)$

При $\lambda=\lambda^{*}$ возникает переход от упорядочения к хаосу. Отношение $\mathrm{x}_{\mathrm{n}} / \mathrm{x}_{\mathrm{n}}{ }^{*}$ характеризует границы изменения управляющего параметра, вплоть до которого величина $\lambda=\lambda *$ сохраняется постоянной. Предположим, что $1 / \lambda *=\Delta_{\mathrm{i}}$. мера устойчивости системы, а параметр $1 /\left(1-\mathrm{x}_{\mathrm{n}}{ }^{*}\right)$ определяет способность системы к перестройкам при $1 / \lambda^{*}=$ const. Тогда для иерархического ряда иррациональных чисел функция самоподобия может быть представлена в виде степенной зависимости: $F=\Delta_{i}^{1 / m}$,

где $\mathrm{m}-$ двоичный код, связанный с оператором действия $\mathrm{m}=2 \mathrm{p}+1$. Здесь $\mathrm{m}=1,2,4,8, ., \mathrm{m}^{*}$, где $\mathrm{m} *$ является порогом самоподобной связи $\Delta_{\mathrm{i}}$ и $\mathrm{m}$.

Адаптивность системы удобно представить как $A_{m}=\lambda_{i}^{*} / \lambda_{i+1}^{*}$ 
Тогда алгоритм самоорганизации устойчивости структур будет иметь вид:

$\mathrm{x}_{\mathrm{n}} * / \mathrm{x}_{\mathrm{n}+1} *=\Delta_{\mathrm{i}}{ }^{1 / \mathrm{m}}$,

где $\Delta_{\mathrm{i}}$ - мера устойчивости самоорганизующейся структуры: $\mathrm{m}$ показатель периодичности перестроек ( $\mathrm{m}=2,4,8,16, \ldots \infty)$ [Иванова В.С., 1992; Иванов О.П., Иванова В.С., 2003]. При $\Delta_{\mathrm{i}}=1 / \delta=0,213$ и $\mathrm{m} \longrightarrow \infty$, отношение $\mathrm{x}_{\mathrm{n}} * / \mathrm{x}_{\mathrm{n}+1} * \longrightarrow\left(\mathrm{x}_{\mathrm{n}}{ }^{*} / \mathrm{x}_{\mathrm{n}+1} *\right)_{\max }$ является пределом адаптивности системы $\mathrm{A}_{\mathrm{m}}{ }^{\max }=0,99$ (при $\mathrm{m} \cong 128$ ). В реальных примерах оценка качества адаптируемости системы может быть проведена за счет решений обобщённого уравнения золотой пропорции: $\mathrm{X}^{\mathrm{P}+1}-\mathrm{X}^{\mathrm{P}}-1=0$

Положительные и отрицательные корни его связаны следующей зависимостью: $\mathrm{d}_{\mathrm{p}}-1=\Delta_{\mathrm{i}}$ 7)

Спектр $\Delta_{\mathrm{i}}$ как мера устойчивости структур будет иметь вид: $0.618 ; 0.465$; $0.380 ; 0.324 ; 0.213$; при $\mathrm{p}=1,2,3,4$.

Отметим, что $1 / \delta=1 / 4,669=0,213$, а устойчивость систем контролируется величиной $\Delta_{\mathrm{i}}=0.618$. Системы с $0,213 \leq \Delta_{\mathrm{I}} \leq 0,618$ являются переходными. Это позволило условие самоуправляемого синтеза структур представить в виде базового алгоритма: $A_{m}=\lambda_{i}^{*} / \lambda_{i+1}^{*}=\mathrm{F}=\Delta_{\mathrm{i}}^{1 / 2 p-1}$

Алгоритм тестироваван и позволяет определять сохранность симметрии после преобразования, т.е. определять условие $\Delta_{\mathrm{i}}=$ const с ростом m (табл.1). 
Таблица 1

Значения меры адаптивности Аm системы к нарушению симметрии, связанной функцией самоподобия с мерой устойчивости симметрии $\left(\Delta_{\mathrm{i}}\right)$ и кодом адаптации m системы к нарушению симметрии

\begin{tabular}{|c|c|c|c|c|c|c|c|c|}
\hline \multicolumn{9}{|c|}{$\mathrm{Am}$} \\
\hline \multirow{2}{*}{ i } & \multirow{2}{*}{$\Delta_{\mathrm{i}}$} & \multicolumn{7}{|c|}{$\mathrm{m}$} \\
\hline & & 2 & 4 & 8 & 16 & 32 & 64 & 128 \\
\hline 2 & 0,465 & 0,68 & - & - & - & - & - & - \\
\hline 3 & $\mathbf{0 , 3 8 0}$ & 0,62 & $\mathbf{0 , 7 9}$ & - & - & - & - & - \\
\hline 4 & 0,324 & $\mathbf{0 , 5 7}$ & 0,75 & 0,87 & - & - & - & - \\
\hline 5 & 0,285 & $\mathbf{0 , 5 3}$ & $\mathbf{0 , 7 3}$ & 0,85 & 0,92 & - & - & - \\
\hline 6 & 0,255 & 0,5 & 0,71 & 0,84 & 0,92 & 0,96 & - & - \\
\hline 7 & 0,232 & 0,48 & 0,69 & 0,83 & 0,91 & 0,95 & 0,98 & - \\
\hline 8 & 0,213 & 0,46 & 0,68 & 0,82 & 0,91 & $\mathbf{0 , 9 5}$ & $\mathbf{0 , 9 8}$ & 0,99 \\
\hline
\end{tabular}

Данный подход применим как для диссипативного, так и для кумулятивного вариантов эволюции любой системы, что доказано рядом экспериментальных исследований зарубежных и российских ученых (рис. 1,2).

Например, случай разрушения металлических сплавов под действием циклических нагрузок (диссипативный вариант эволюции) (рис.1). Здесь соотношения между уровнями напряжений $\sigma_{\mathrm{w} 1} / \sigma_{\mathrm{w} 2}=\sigma_{\mathrm{w} 2} / \sigma_{\mathrm{w} 3}$ в точках бифуркации $\left(\tau_{\mathrm{T}} / \sigma_{\mathrm{T}}\right)^{1 / 2}=\Delta_{\mathrm{i}}=0,618$ - универсальная постоянная разрушения материала, где $\tau_{\mathrm{T}}$ - теоретическая прочность на сдвиг, $\sigma_{\mathrm{T}}-$ прочность на отрыв. Для случая алюминиевых сплавов $\Delta_{\mathrm{i}}=0,213$. Подтверждено российскими и японскими исследователями. 


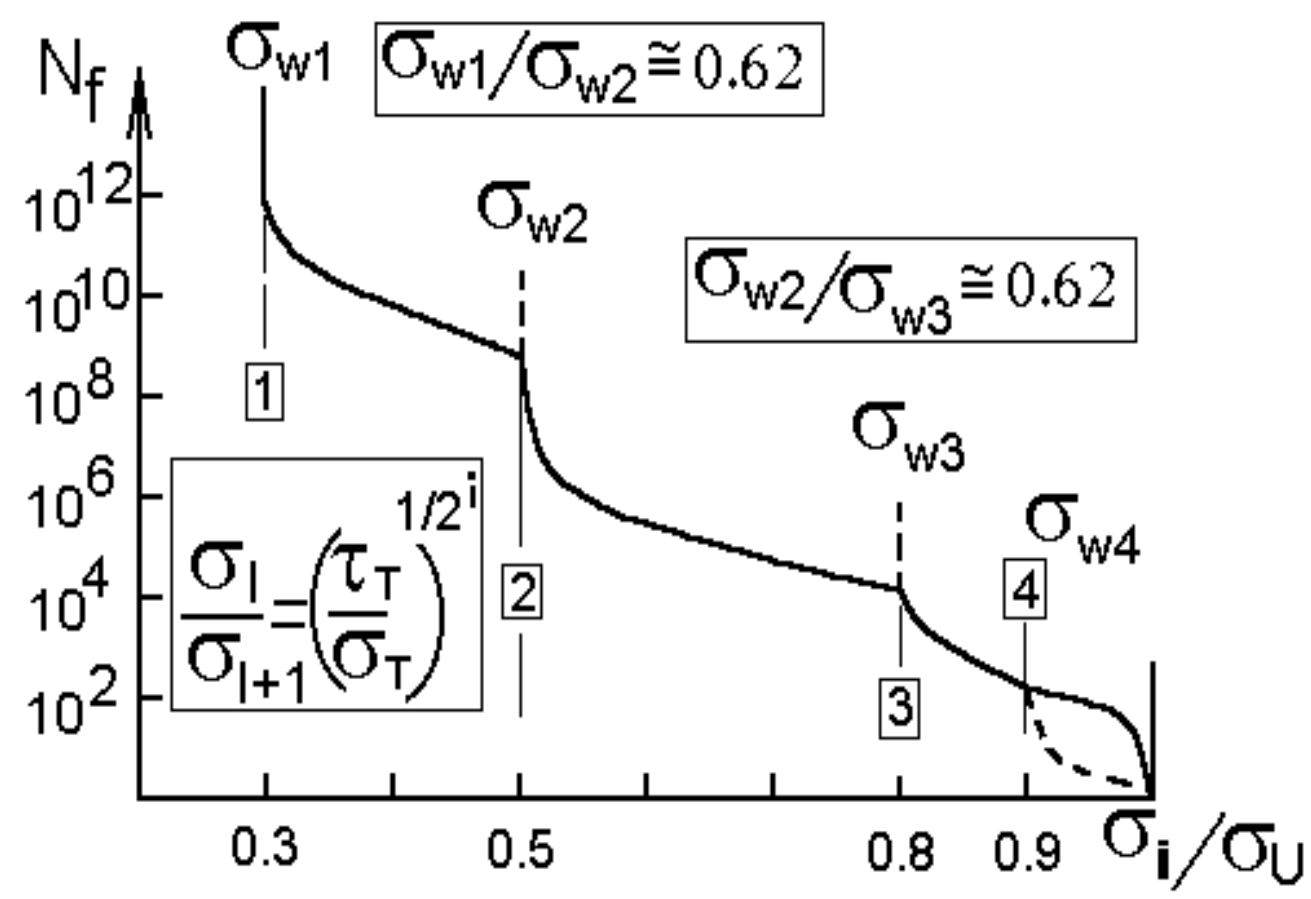

Рис. 1. Бифуркационная диаграмма усталостного разрушения для сплавов на основе железа [Шанявский А.А., 2007]

Для варианта кумулятивной эволюции по флуктуационному или волновому зарождению кристаллов под влиянием ультрадисперсных порошков (УДП) на структурообразование и свойства кальцийфосфатного соединения (КФС) [Седельников В.В.и др., 2005; Седельников В.В., 2007]. Процесс кристаллизации (кумулятивная эволюция) исследовался методом открытой капли. Кристаллизация происходила за счет изменения концентрации раствора, в результате испарения воды в капле.

Экспериментальные данные показывают, что для кристаллизующейся системы, состоящей из раствора КФС + УДП (Тi $\mathrm{C}_{0.3} \mathrm{~N}_{0.7}$ ), отношение управляющего параметра (концентрация УДП) предыдущей $\lambda_{\mathrm{cn}}$ и последующей $\lambda_{\text {Cn+1 }}$ точек бифуркаций во всех концентрационных диапазонах величина постоянная и равна: $F=\frac{\lambda_{c_{n}}^{I}}{\lambda_{c_{n+1}}^{I}}=\frac{\lambda_{c_{n}}^{I I}}{\lambda_{c_{n+1}}^{I I}}=\frac{\lambda_{c_{n}}^{I I I}}{\lambda_{c_{n+1}}^{I I I}}=0,9900596$

Для кристаллизующихся систем мера адаптивности - не только коэффициент самоподобия системы, но и показатель состояния системы. 


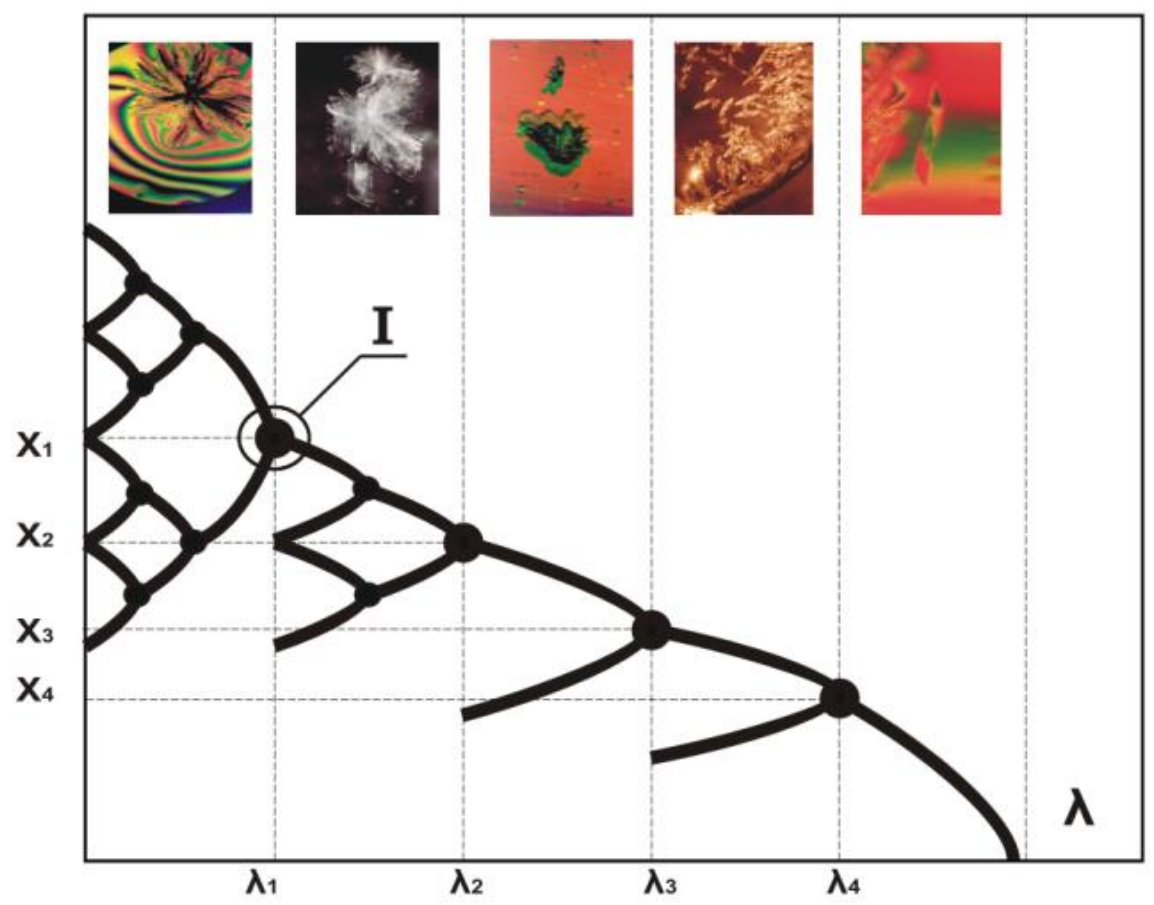

Рис. 2. Бифуркационная диаграмма перестройки кристаллизующихся структур $\left(\lambda_{1} X_{1}\right.$ - переход от сферолитов к слоистым дендритам; $\lambda_{2} X_{2}$ - переход от слоистых к плоским дендритам; $\lambda_{3} X_{3}-$ переход от плоских дендритов к слоистым пластинчатым кристаллам; $\lambda_{4} \mathrm{X}_{4}$ - переход от слоистых к пластинчатым кристаллам)

Диаграмма дает качественную оценку влияния управляющего параметра на тренд структурообразования кристаллизующихся систем (к сферолитам или к пластинчатым формам). Используя бифуркационную диаграмму и табулированные значения $\mathrm{A}_{\mathrm{m}}$ через параметры порядка можно определить в каком структурном состоянии находится кристаллизующаяся система.

Опробование нами универсального алгоритма на 15 крупнейших землетрясениях на территории бывшего СССР показало возможность более точной оценки интенсивности землетрясений вместо субъективной оценки по радиусам изосейст. Реализации этих переходов происходит в точках бифуркаций в наноразмерных объемах, запуская тем самым макропроцесс. Мы специально разнопланово показали широкие возможности данного алгоритма и верим в его существенные перспективы. 


\section{Список литературы}

1. Иванова В.С. Синергетика. Прочность и разрушение металлических материалов. М.: Наука. 1992, с. 44.

2. Иванов О.П., Иванова В.С. Нелинейная дискретная сейсмология в свете универсальных хаотических динамических систем. Сб. «Синергетика.» T5.C.128-151.

3. Седельников В.В. Особенности флуктуационного зарождения кристаллов. Металлургия Машиностроения. 2007, №6, с.27-29.

4. Шанявский А.А. Моделирование усталостных разрушений металлов. Синергетика в авиации. Уфа. «Монография». 2007. 499 с. 